\title{
Assays with Commercially Produced Trichogramma (Hymenoptera: Trichogrammatidae) To Determine Suitability for Obliquebanded Leafroller (Lepidoptera: Tortricidae) Control
}

\author{
D. S. LAWSON, J. P. NYROP, AND W. H. REISSIG \\ Department of Entomology, Cornell University, New York State Agricultural Experiment Station, \\ Geneva, NY 14456
}

\begin{abstract}
Environ. Entomol. 26(3): 684-693 (1997)
ABSTRACT Laboratory assays were used to compare the ability of commercially produced Trichogramma spp. to parasitize eggs of the obliquebanded leafroller, Choristoneura rosaceana (Harris), in the laboratory and field. Trichogramma platneri Nagarkatti parasitized more obliquebanded leafroller eggs per egg mass than did Trichogramma pretiosum Riley or Trichogramma minutum Riley produced by either of 2 insectaries. T. minutum produced at 1 insectary caused significantly more host mortality by host feeding and repiercing than by parasitism. Variation in parasitoid performance from different insectaries and among shipments from the same insectary was common. Young egg masses were more heavily parasitized than old egg masses. Parasitism increased as the number of conspecific female Trichogramma spp. placed on the same host increased, but the number of eggs parasitized per female decreased. Exposure of host egg masses to female Trichogramma spp. prevented additional oviposition in the same egg mass by conspecific, ovipositionally experienced females but had no effect on parasitism by conspecific, ovipositionally naive females. The method used to attach sentinel egg masses to foliage influenced parasitism rates. Moistening the leaf with water and adhering the egg mass to the moistened leaf had the least impact. Inundative releases of Trichogramma spp. into an apple orchard paralleled laboratory assays by showing greater parasitism of obliquebanded leafroller egg masses by $T$. platneri than with $T$. minutum. However, extreme differences observed in field performance between the 2 species was not predicted from the assays.
\end{abstract}

KEY WORDS Choristoneura rosaceana, Trichogramma spp., apple, biological control

OBLIQUEBANDED LEAFROLLER, Choristoneura rosaceana (Harris), is a major pest of apples in New York State. In some orchards, fruit damage from this pest can reach $20 \%$, even when multiple insecticide applications are used for control (Lawson et al. 1996). One alternative control strategy being investigated for this pest is inundative release of commercially produced egg parasitoids of Trichogramma spp. Casual assessments in insecticidetreated commercial orchards have revealed as many as $40 \%$ of the obliquebanded leafroller egg masses parasitized by endemic Trichogramma spp., with $\approx 50$ parasitized eggs per located egg mass.

Hassan (1990) suggested that when selecting Trichogramma spp. for augmentative release, attributes which directly influence field performance, such as searching ability (habitat location, host location), host preference (recognition, acceptance, suitability), and tolerance to environmental conditions, should be evaluated. Hassan (1994) outlined a 3-step procedure for assessing the suitability of Trichogramma spp. First, the contact and parasitism method for evaluating host preference was recommended to screen host suitability and preference of Trichogramma spp. quickly in the laboratory. The Trichogramma spp. that indicate high levels of host acceptance would be examined under semifield conditions to assess searching capacity. Finally, Trichogramma spp. possessing good searching ability would then be examined under field conditions to assess efficacy.

We modified the method proposed by Hassan (1994) to identify I of the many commercially produced Trichogramma spp. which could be tested in an inundative release program. We examined the following 4 factors thought to influence the ability of Trichogramma spp. to parasitize obliquebanded leafroller egg masses and therefore could be used to rank field performance: (1) host acceptance and the effect of host age on parasitism, (2) the effect of multiple conspecific female parasitoids on the same host, (3) the effect of exposing hosts to conspecific females on subsequent parasitism by another female, and (4) the ability of parasitoids to locate and parasitize hosts on small potted apple trees with and without provisioning. 
Results of these assays were compared with those obtained from inundative releases of Trichogramma spp. into an apple orchard.

\section{Materials and Methods}

Trichogramma minutum Riley, T. platneri Nagarkatti, and T. pretiosum Riley produced by Rincon-Vitova insectary (Ventura, CA) and T. minutum produced by Bio-Logicals insectary (CIBA-GEIGY, Guelph, Ontario, Canada) were examined as potential parasitoids of obliquebanded leafroller egg masses. T. minutum produced by Rincon-Vitova and Bio-Logicals are indicated with (Rv) and (Bl), respectively. As part of the quality control procedures at these insectaries, specimens were periodically sent to an expert taxonomist for identification. We relied on these identifications to ensure our studies were conducted with the appropriate species. Parasitoids were shipped by overnight delivery to Geneva, NY, where all studies were conducted. Trichogramma spp. from RinconVitova insectary were shipped as pupae in parasitized eggs of Angoumois grain moth, Sitotroga cercalella (Olivier), and those from Bio-Logicals insectary were in parasitized eggs of Mediterranean flour moth, Ephestia kuehniella Zeller, glued to cardboard. Upon receipt, strips of cardboard containing $\approx 10,000$ parasitized eggs were placed into polystyrene snap cap vials ( 25 by $50 \mathrm{~mm}$ ) and incubated at $25: 23^{\circ} \mathrm{C}, 75 \% \mathrm{RH}$, and a photoperiod of 16:8 (L:D) h. Vials were checked daily for emerging adults, and assays were initiated $24 \mathrm{~h}$ after 1st adult emergence.

Obliquebanded leafroller egg masses were collected from a laboratory colony reared on fava beans (Glass and Hervey 1962). Male and female moths were placed inside plastic bags with moist cotton and held at room temperature and ambient light conditions. Egg masses were deposited on the plastic bags, and each morning new egg masses were circled with a colored marker to indicate age. Appropriately aged egg masses were collected by gently peeling them off the plastic with a probe and small paint brush. Egg masses ranged in size from $\approx 70$ to 350 eggs per egg mass. Female Trichogramma spp. do not oviposit >50 eggs in a $24-\mathrm{h}$ period; therefore, the number of available hosts in assays was never limiting (Houseweart et al. 1983, Yu et al. 1984a).

Parasitiom by Trichogramma spp. and Effect of Host Age. One 24-h-old gravid female parasitoid was placed in a vial containing 1 egg mass that was either $24,48,72$, or $96 \mathrm{~h}$ old, and parasitism and host mortality were determined after $24 \mathrm{~h}$. Twenty vials containing eggs of each age were established for each of 3 separate shipments of each species. Ten vials had a parasitoid added to them and 10 vials served as controls. Vials were placed in the growth chamber previously described and after $24 \mathrm{~h}$, females were removed and assessed as alive or dead. Egg masses were then incubated un- til parasitized eggs became visible. All parasitized eggs in each egg mass were counted, and the egg masses were returned to the incubator until obliquebanded leafroller larvae hatched. Larvae were counted as they hatched and removed to prevent cannibalism of the egg mass. The numbers of wasps emerging from parasitized eggs were counted and their sex recorded. All obliquebanded leafroller eggs that failed to hatch also were counted. This procedure also was used to count the number of larvae and unhatched eggs in control vials. To ensure that gravid female parasitoids were used, only parasitized egg masses producing $\geq 1$ female offspring were included in analyses ( $\mathrm{Yu}$ et al. 1984a). Although provisioning has been shown to increase longevity and fecundity (Ashley and Gonzalez 1974, Yu et al. 1984a), we chose not to provision the parasitoids in this study. We expected this to aid in distinguishing effectiveness among the species and to indicate which parasitoids were of higher quality, particularly between the 2 insectaries.

Host mortality other than parasitism (stings and host feeding) was estimated by subtracting percentage parasitism from corrected total mortality, calculated using Abbott's (1925) formula. Data were transformed using $\log (x+1)$ and analysis of variance (ANOVA) was applied. The ANOVA model included parasitoid species (insectary), a shipment factor which allowed for examination of parasitoid performance among different shipments, interactions, and a covariate that indicated whether the parasitoid was alive or dead at the end of the 24-h exposure. Means were separated using the Fisher protected LSD (Abacus Concepts 1989).

A method of determining parasitism levels without counting all eggs per egg mass would greatly reduce the time necessary to evaluate parasitism. Toward identifying such a method, Pearson correlations were used to determine the relationship between number and percentage of obliquebanded leafroller eggs parasitized per egg mass, and between the number of eggs parasitized and the number of eggs per egg mass. Data from all species and age classes were used.

Multiple Trichogramma Females per Egg Mass. Only T. minutum (Rv) and T. platneri were used in this study because they caused the greatest parasitism level of all Trichogramma spp. examined (previous assay). Twenty-four-hour-old egg masses (24 h old) were exposed to $1,2,3$, or 424 -h-old conspecific female parasitoids simultaneously for $24 \mathrm{~h}$ in a vial. The number of females alive or dead was assessed at the end of the exposure. This study was conducted under the same conditions as the 1st assay with the exception that thin streaks of honey were placed inside the vials to reduce mortality of the parasitoids. Parasitism was evaluated as previously described. The study was conducted with 7 different shipments of each parasitoid species and 3-7 replicates of each treatment per shipment. 
Individual female Trichogramma oviposition rates were calculated for all egg masses and for only egg masses that had been parasitized. Data were transformed using $\log (x+1)$ and the effect of species, number of females per egg mass, shipment, and interactions were examined using ANOVA and linear contrasts (Abacus Concepts 1989).

Effect of Previous Host Visits. Twenty vials, each containing honey streaks, had one 24-h-old female Trichogramma spp. placed in them. Into 15 of the vials, one 24-h-old obliquebanded leafroller egg mass also was placed. All vials were held under previously described conditions for $24 \mathrm{~h}$. After 24 $h$, vials were examined for dead or living parasitoids which were removed. The following 3 treatments were used: (1) (previous exposure) the females from 5 vials were placed individually onto fresh 24-h-old egg masses in new vials; (2) (previous exposure + experienced) into each of 5 vials containing a visited egg mass, 1 experienced female which had been confined with an egg mass for the previous $24 \mathrm{~h}$ was placed; (3) (previous exposure + inexperienced) into another group of 5 vials containing a visited egg mass, 1 naive female which had been held in a vial with just honey was placed. The remaining 5 egg masses were not exposed to additional parasitoids. All vials were held under previously described conditions for an additional $24 \mathrm{~h}$, at which time all parasitoids were removed and rated as dead or alive. Parasitism was assessed by counting the number of parasitized eggs per egg mass. This experiment was performed with 6 different shipments of $T$. platneri and $T$. minutum $(\mathrm{Rv})$ and 5 replicates of each treatment.

Data were subjected to an ANOVA with treatment factors, a shipment factor which allowed for the examination of parasitoid performance among different shipments, an interaction term, and a covariate which indicated whether the Trichogramma female was alive or dead at the end of the assay ( $48 \mathrm{~h}$ ). Means were separated using the Fisher protected LSD (Abacus Concepts 1989). Parasitism rates caused by the same female during 2 consecutive exposures to 24-h-old egg masses were compared using a paired $t$-test.

Trichogramma spp. Searching Ability and Effect of Provisioning. To determine the most appropriate method for attaching sentinel egg masses to apple leaves for use in the following assay and for later use in the field, individual 24-hold egg masses were attached to an apple leaf by affixing a small piece of double sided tape to the apple leaf and placing an egg mass on it; cutting an egg mass out of the plastic rearing bag, and pinning the plastic to the leaf; and moistening the leaf with a small paint brush dipped in distilled water, then placing the egg mass on the leaf. Individual leaves with egg masses affixed were placed in individual vials. A small hole in the cap of the vial allowed the leaf petiole to protrude through the cap into moist cotton. On the midrib of the leaf a small streak of honey was placed. Into each vial, 1 mated female Trichogramma was placed. Two shipments of T. platneri and T. minutum (Rv) were tested, and 10 replicates per treatment per shipment were completed. All vials were held under previously described conditions for $24 \mathrm{~h}$, at which time females were removed and rated as alive or dead. A small piece of leaf containing the egg mass was removed and placed in a vial and incubated until parasitism could be assessed.

Data were transformed as $\log (x+1)$ and subjected to an ANOVA with treatment factors, a shipment factor which allowed for an examination of parasitoid performance among different shipments, interactions, and a covariate which indicated if the female parasitoid was alive or dead at the end of each assay. Means were separated using the Fisher protected LSD (Abacus Concepts 1989).

Eight screened cages ( 1 by $1 \mathrm{~m}$ ) each containing 3 apple seedlings $(0.5 \mathrm{~m}$ tall) with an average of 7.8 leaves were placed in a large growth chamber with a photoperiod of $16: 8$ (L:D) h, $25: 23^{\circ} \mathrm{C}$, and $75 \% \mathrm{RH}$. Onto one of the three seedlings in each cage, one $24 \mathrm{~h}$ old egg mass was attached to the top of a midcanopy leaf by moistening the leaf with distilled water and placing the egg mass on the leaf. This method of egg mass attachment had the least effect on parasitism levels. Into each of 4 cages, 60 female parasitoids were released from a glass Pasteur pipette. Into the other 4 cages, females were released from glass Pasteur pipettes provisioned with honey: After $72 \mathrm{~h}$, all egg masses were collected and placed in individual vials and incubated as previously described until parasitism could be assessed. Each of the pipettes were examined after $72 \mathrm{~h}$, and all parasitoids remaining were counted. This assay was conducted with 5 shipments of T. platneri and T. minutum (Rv), and apple seedlings were randomized before being placed in cages.

Data were transformed using $\log (x+1)$ and subjected to an ANOVA with treatment factors, a shipment factor which allowed for an examination of parasitoid performance among different shipments, interactions, and a covariate consisting of the number of parasitoids remaining in the release container at the end of each assay. Means were separated using the Fisher protected LSD (Abacus Concepts 1989).

Parasitiom of Obliquebanded Leafroller Egg Masses by Inundatively Released Trichogramma spp. To examine how T. platneri and T. minutum (Rv) performed under field conditions, inundative releases were made into 4 plots (0.14 ha) of apple trees. Plots contained 'Red Delicious' and 'McIntosh' cultivars planted at a density of 1,000 trees per hectare, with an average tree height of $3-4 \mathrm{~m}$. All plots were separated by $\geq 30 \mathrm{~m}$. In the middle of each plot, 5 adjacent trees within the same row and the 2 trees in the row adjacent to the middle tree each had one 24-h-old obliquebanded leafroller egg mass placed in them. All sentinel egg masses were placed at midcanopy height near the 
center of the tree on the upper side of a leaf by moistening a leaf with distilled water and placing the egg mass on the moistened leaf. Sentinel egg masses were collected and new 24-h-old egg masses were placed in the field every $2 \mathrm{~d}$ for $8 \mathrm{~d}$ after parasitoid release. Care was taken not to place new egg masses on leaves previously occupied by a sentinel egg mass.

Cards containing Trichogramma spp. were cut into sections containing $\approx 5,000$ parasitized eggs and placed individually in vials $(25$ by $50 \mathrm{~mm})$ that had been provisioned with honey. Vials were held in growth chambers as previously described. Twenty-four hours after the first adult emerged, vials were transported to the field, and 4 vials were attached to the lowest scaffold branch $(\approx 0.5-1 \mathrm{~m}$ above ground) of the middle tree of 2 plots. Caps were removed from the vials and fine mesh placed over the opening to prevent predation of the egg card. One additional vial was placed in each plot and remained capped; it was used to determine sex ratio, percentage brachypterous individuals, and number of parasitoids released. Treatment and control plots were randomized between releases. T. minutum (Rv) was released on 9 and 22 August, whereas $T$. platneri was released on 25 July and 6 September 1995. The 2-wk interval between releases was sufficient for most parasitoids to have died or dispersed from the plots. If some individuals from previous releases remained in the plots, parasitism in the control plots would account for this, and comparison of treatment to control plots would indicate parasitism levels from the inundative release being examined.

All data were transformed using $\log (x+1)$ and subjected to a repeated measures ANOVA with a parasitoid species factor, an interaction between time and parasitoid species, and a blocking factor which accounted for variability in parasitoid shipment and different environmental conditions during each release (Abacus Concepts 1989).

\section{Results and Discussion}

Parasitism by Trichogramma spp. and Effect of Host Age. In this assay, we measured obliquebanded leafroller egg parasitism and mortality attributed to host feeding and repiercing (Manweiller 1986) by different Trichogramma spp. We also determined the effect of host age on parasitism. Trichogramma spp. have different host preferences which may be inherent or influenced by the host on which they were reared (Smith et al. 1986; Hassan 1989, 1990). Host age can have a pronounced effect on parasitism levels, with younger eggs generally more heavily parasitized than older eggs (Houesweart et al. 1982, Yu et al. 1984a). To be effective when released, a species of Trichogramma must be able to parasitize the host and ideally, parasitism rates would be little affected by host age.
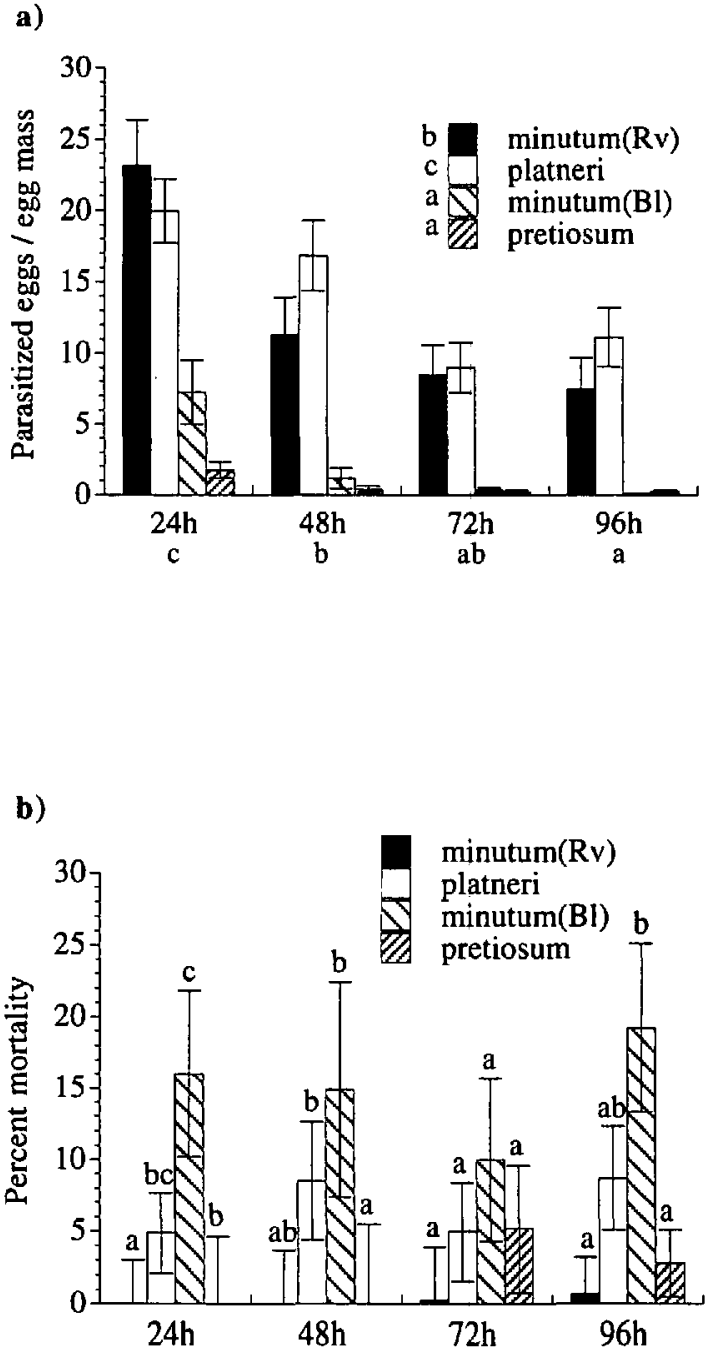

Fig. 1. (a) Parasitism of 4 obliquebanded leafroller egg mass age classes by gravid $T$. platneri, T. pretiosum, and $T$. minutum produced at 2 different insectaries [(BI) and (Rv)] and calculated from only parasitized egg masses. Trichogramma spp. names preceded by the same letter in the legend do not have significantly different parasitism rates. Host age class sharing the same letter do not have significantly different parasitism rates. (b) Percentage mortality of 4 obliquebanded leafroller egg mass age classes caused by $T$. platneri, T. pretiosum, and $T$. minutum produced at 2 different insectaries $[(\mathrm{Bl})$ and (Rv)] from factors other than parsitism. Histograms within the same age class sharing the same letter are not significantly different.

Trichogramma platneri caused the greatest parasitism, T. minutum (Rv) caused an intermediate level of parasitism, and T. pretiosum and T. minutum $(\mathrm{Bl})$ parasitized similar and the fewest eggs ( $F$ $=69.2 ; \mathrm{df}=3,456: P \leq 0.001$ ) (Fig. la). Twentyfour-hour-old egg masses were most heavily parasitized and parasitism declined as host age increased $(F=16.8 ; \mathrm{df}=3,456 ; P \leq 0.001)$. Death of female Trichogramma spp. during the course of 
the assay significantly reduced parasitism levels $(F$ $=9.60 ; \mathrm{df}=1,456 ; P=0.002)$. However, no interaction between Trichogramma spp. and female death was found, indicating differences among Trichogramma spp. parasitism rates are not the result of females dying during the 24-h exposure period.

Mortality caused by host feeding and repiercing revealed a significant species $\times$ host age interaction $(F=2.16$; df $=9,456 ; P=0.024)$. Therefore, separate ANOVAs were conducted for each host age. Significant differences among Trichogramma spp. and source were observed for $24-h, 48-h$, and 96-h-old egg masses $(24 \mathrm{~h}: F=9.37$; df $=3,113$; $P \leq 0.001$. 48 h: $F=7.17 ; \mathrm{df}=3,111 ; P \leq 0.001$. $96 \mathrm{~h}: F=4.52 ; \mathrm{df}=3,112 ; P=0.005$ ) (Fig. $1 \mathrm{~b}$ ). In general, $T$ minutum $(\mathrm{Bl})$ caused the greatest mortality followed by $T$. platneri. The lowest mortality from factors other than parasitism was caused by $T$. minutum (Rv) and T. pretiosum, which produced similar levels. Significant shipment effects were observed when mortality other than parasitism was assessed, indicating variable parasitoid quality among shipments from the insectaries $(F=$ 7. $60 ; \mathrm{df}=2,455 ; P \leq 0.001$ ).

Differences in parasitism by different Trichogramma spp. exposed to the same host have been reported (Hassan 1989). It also has been shown that the same Trichogramma spp. collected from different areas and exposed to the same host show different parasitism levels (Smith et al. 1986), indicating that external factors can influence host acceptance. In our study, T. minutum (Rv) was reared on S. cerealella eggs and T. minutum (BI) was reared on E. kueniella eggs.

Different levels and types of host mortality caused by T. minutum (Rv) and T. minutum (BI) were observed. T. minutum (Rv) caused significantly greater direct parasitism, whereas T. minutum (Bl) caused significantly greater mortality in ways other than direct parasitism, likely through host feeding and repiercing (Manweiller 1986). T. platneri was reported to cause mortality to cabbage looper, Trichoplusia ni (Hübner) eggs by host feeding and repiercing, whereas T. minutum did not host feed or repierce (Manweiller 1986). Our results with these same parasitoid species indicate that T. minutum (Bl) caused host mortality to obliquebanded leafroller eggs by host feeding and repiercing, whereas $T$. platneri causes mortality by parasitism.

This assay clearly demonstrated the profound effect that different commercial insectaries or rearing methods or both can have on the same parasitoid species. Differences in performance of $T$. minutum (Rv) and T. minutum (Bl) probably result from different rearing conditions at the 2 commercial insectaries and the use of different hosts.

The pronounced effect of host age on parasitism by Trichogramma spp. has been reported (Houseweart et al. 1982, Yu et al. 1984a, Pak 1986). However, parasitoid/host ratio can influence host acceptance, with parasitism of older hosts occurring more frequently at high parasitoid/host ratios (Pak 1986). In our study, a low parasitoid/host ratio was used. Therefore, we expected our results to show large differences in host acceptance by the Trichogramma spp. examined.

Inhibition of female Trichogramma oviposition coincides with blastokiness of the host egg (Pak 1986). Eggs may be defended from parasitoids by rapid development which uses nutrients needed by the developing parasitoid, thereby preventing its development (Ruberson et al. 1987). In our study, it appears that either female Trichogramma spp. are choosing not to oviposit in older eggs, or oviposition is the same in all host age classes but parasitoid survival is reduced in older hosts. A combination of the 2 events is probably occurring. Female parasitoids have been shown to discriminate between hosts and predominantly oviposit into those providing the best resources for her progeny (Schmidt 1994). However, we observed a significant $(F=2.89 ; \mathrm{df}=3,456 ; P=0.035$ ) host age effect on mortality other than parasitism, apparently caused by greater host mortality in older egg masses (Fig. 1b). If oviposition is the same among all host ages but eggs do not have enough time to develop in older hosts, the action of penetrating the chorion during oviposition may kill the egg, resulting in the observed trend of greater mortality in older egg masses caused by means other than direct parasitism.

It is desirable for inundatively released parasitoids to cause mortality to host eggs through parasitism because offspring from parasitized eggs will cause subsequent parasitism under field conditions. T. minutum (Rv) and T. platneri caused the greatest parasitism levels of all Trichogramma spp. and sources examined. Therefore, only these 2 species were used in subsequent assays.

Data from this study were used to determine the. most appropriate way to evaluate parasitism. A significant relation between the number and percentage parasitized eggs per egg mass $(r=0.858$, $P \leq 0.001)$ was found; however, no relation $(r=$ $-0.026, P=0.568$ ) between the mean number of eggs per egg mass and number of parasitized eggs per egg mass was found. Therefore, in the ramaining studies, we chose to use the number of eggs parasitized per egg mass as a measure of parasitism because counting the number of parasitized eggs is easier than determining percentage parasitism in each egg mass. Similar observations were made for the relation between the number of $T$. minutumparasitized spruce budworm eggs and the number of eggs per egg mass (Bai and Smith 1994).

Multiple Trichogramma Females per Egg Mass. In this assay, we measured parasitism levels obtained from exposing an egg mass to single verses multiple parasitoid females. With the large number of obliquebanded leafroller eggs per egg mass available for parasitism, and the large number of parasitoids present during inundative releases, 
a)

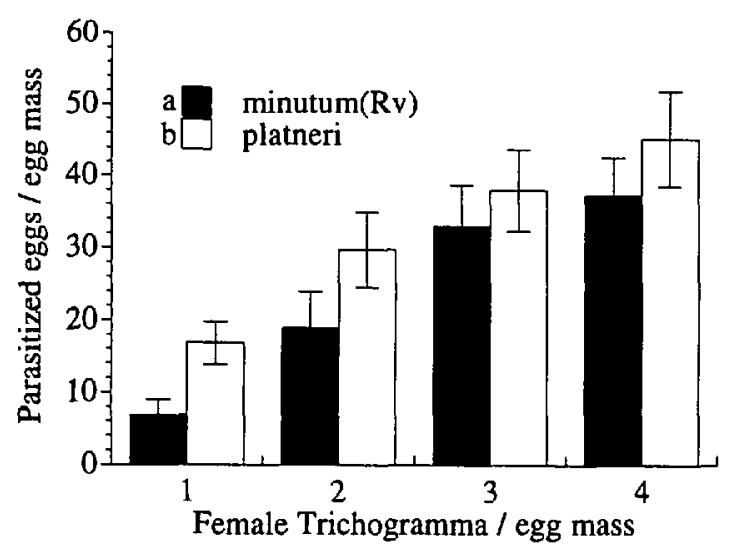

b)

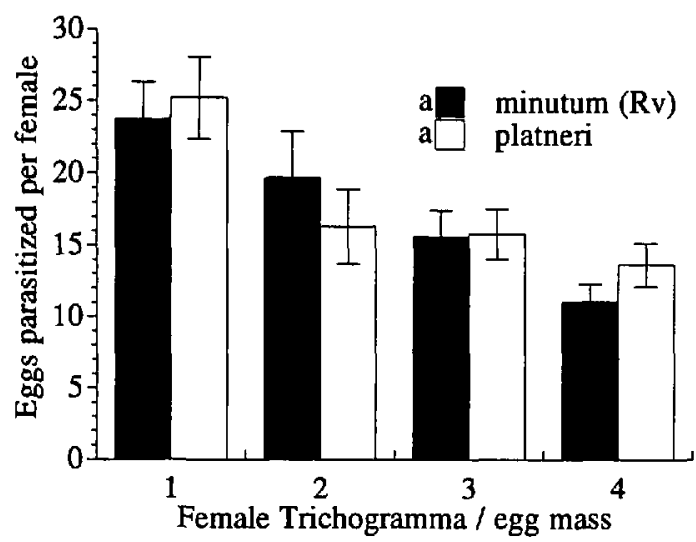

c)

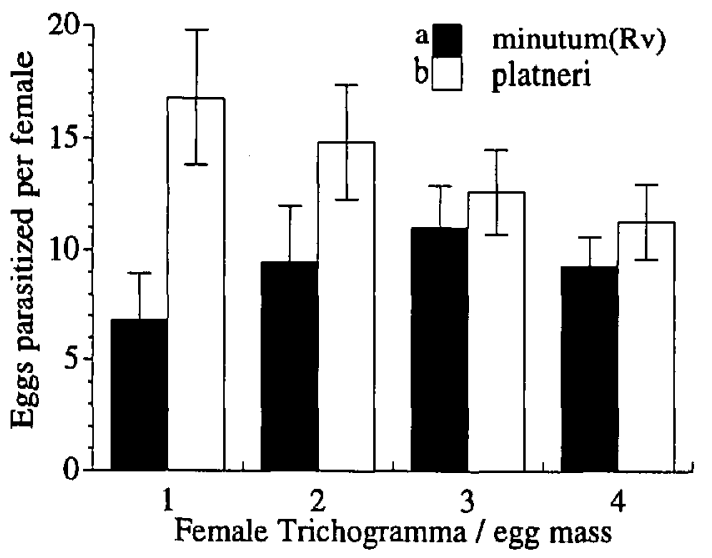

Fig. 2. (a) Parasitism of obliquebanded leafroller egg masses when 4 densities of conspecific female Trichogramma sp. were placed simultaneously on 1 egg mass; calculations included all egg masses. Trichograimma spp. names preceded by the same letter in the legend do not have significantly different parasitism rates. (b) Parasitism per female Trichogramma when 4 densities of conspecific females were placed simultaneously on 1 egg mass; cal-
$>1$ female Trichogramma may parasitize an egg mass simultaneously. Yu et al. (1984a) reported that multiple parasitoids per host caused some level of interference in parasitism levels not observed when a single female was present. The determination of how changing numbers of female Trichogramma per host affects total parasitism and parasitism per individual female may contribute to our ability to distinguish among Trichogramma spp. for their suitability as parasitoids of obliquebanded leafroller eggs.

Parasitism, calculated from all eggs, whether parasitized or not, increased as the number of conspecific female Trichogramma per egg mass increased ( $F=28.15 ; \mathrm{df}=1,186 ; P \leq 0.001$ ) (Fig. 2a). T. platneri parasitized significantly more eggs per egg mass than T. minutum (Rv) at all parasitoid densities $(F=9.03 ; \mathrm{df}=1,186 ; P=0.003$ ). As the number of females per egg mass increased, the number of eggs parasitized (calculated only from parasitized egg masses) by each ovipositing female declined $(F=12.54$; $\mathrm{df}=1,123 ; P \leq 0.001$ ) (Fig. 2b). No differences between T. minutum (Rv) and $T$. platneri were recorded. The decline in parasitism per ovipositing female as female density increased may indicate interference among females when $>1$ is present. When parasitism per female was calculated from all egg masses, whether parasitized or not, significantly $(F=10.21$; df $=1$, $186 ; P \leq 0.002$ ) fewer eggs were parasitized by $T$. minutum compared with $T$. platneri with no interaction between species and female density (Fig. $2 c$ ). This appears to be the result of a greater proportion of T. platneri females ovipositing (79.4\%) compared with $T$. minutum (57.1\%). A decline in the level of parasitism per female T. minutum as the number exposed to eggs of codling moth, $C y$ dia pomonella (L.), increased also has been reported (Yu et al. 1984a).

Parasitism levels were significantly $(F=5.12$; df $=6,123 ; P \leq 0.001)$ different among the shipments received from the insectary, ranging from an average of 3.8 to 20.4 parasitized eggs per egg mass. This likely resulted from variation in the commercial production of these parasitoids (Newton 1993).

Parasitism rates in this study were much lower than those recorded in the Trichogramma spp, and host age study for the same Trichogramma spp. For example, in the previous study, T. minutum

culated from only parasitized egg masses. Trichogramma spp. names preceded by the same letter in the legend do not have significantly different parasitism rates. (c) Parasitism per female Trichogramma sp. when 4 densities of conspecific females were placed simultaneously on 1 egg mass; calculated from both parasitized and unparasitized egg masses. Trichogramma spp. names preceded by the same letter in the legend do not have significantly different parasitism rates. 
Table 1. Parasitism of obliquebanded leafroller egg masses, previously visited by conspecific female Trichogramma spp., exposed to ovipositionally experienced or inexperienced Trichogramma spp. females

\begin{tabular}{|c|c|c|c|}
\hline \multirow[b]{2}{*}{ Species } & \multicolumn{3}{|c|}{$\begin{array}{l}\text { Mean no. of parasitized } \\
\text { eggs per egg mass }\end{array}$} \\
\hline & $\begin{array}{c}\text { Previous } \\
\text { exposure } \\
\text { (SE) }\end{array}$ & $\begin{array}{l}\text { Previous } \\
\text { exposure }+ \\
\text { experienced } \\
(\mathrm{SE})\end{array}$ & $\begin{array}{c}\text { Previous } \\
\text { exposure }+ \\
\text { inexperienced } \\
\text { (SE) }\end{array}$ \\
\hline T. platneri & $14.0(2.7) \mathrm{a}$ & $17.1(3.7)_{\mathrm{a}}$ & $26.2(4.5) b$ \\
\hline T. minutum $(\mathrm{Rv})^{a}$ & $13.9(2.2) \mathrm{A}$ & $18.3(2.7) \mathrm{A}$ & $26.3(3.4) \mathrm{B}$ \\
\hline
\end{tabular}

Analyses were performed separately for euch Trichogramma spp. Means followed by the same letter are not significantly different (Fisher protected LSD, $P>0.05$ ).

a $T$. minutum produced at Rincon-Vitova insectary (Ventura, CA)

(Rv) and T. platneri parasitized between 20 and 23 eggs per egg mass; however, in the current study, the same species parasitized between 8 and 18 eggs per egg mass. Although these studies were conducted at different times, conditions were similar. However, in the current study, all vials were provisioned with honey. Therefore, we hypothesize that female Trichogramma spp. spent time feeding and not ovipositing in the current study, resulting in lower parasitism rates.

Effect of Previous Host Visits by Female Trichogramma spp. In this assay, the effect of a previous host visit on parasitism rates by ovipositionally naive or experienced females exposed to the same egg mass was measured. Obliquebanded leafroller egg masses contain more eggs than $1 \mathrm{fe}-$ male Trichogramma spp. can parasitize. Therefore, under field conditions, many eggs in an egg mass remain unexploited even after parasitism by a female Trichogramma sp. Host marking by conspecific Trichogramma spp. has been shown to prevent successive oviposition into solitary eggs by ovipositionally experienced females. But the effect of host marking on parasitism of egg masses is unknown (Salt 1937, Klomp et al. 1980).

Addition of an ovipositionally inexperienced female Trichogramma to an obliquebanded leafroller egg mass previously visited by a conspecific female significantly $(F=8.67 ; \mathrm{df}=2,194 ; P \leq 0.001)$ increased parasitism in both species (Table 1); however, the addition of an ovipositionally experienced female did not. One explanation is that experienced females depleted their egg supply during their 1st host visit. We found that $T$. platneri and $T$. minutum (Rv) parasitized the same number of eggs during their 1st and 2nd exposure to fresh hosts (T. platneri: $t=2.02, \mathrm{df}=20, P=0.057 ; T$. minutum (Rv): $t=1.442, \mathrm{df}=22, P=0.163$ ) (Table 2).

It was expected that the total number of parasitized eggs from the lst and 2nd exposure would equal that of the 1 exposure plus an experienced female if no inhibition occurred. This was not observed (Table 1). However, total parasitism for
Table 2. Parasitism of obliquebanded leafroller egg masses by the same Trichogramma sp. female during consecutive exposures to unexposed 24-h-old obliquelanded leafroller egg masses

\begin{tabular}{lccc}
\hline \hline & \multicolumn{3}{c}{ Mean no. parasitized eggs per egg mass } \\
\cline { 2 - 4 } Species & $\begin{array}{c}\text { 1st } \\
\text { exposure } \\
\text { (SE) }\end{array}$ & $\begin{array}{c}\text { 2nd } \\
\text { exposure } \\
\text { (SE) }\end{array}$ & $\begin{array}{c}\text { Total } \\
\text { for both } \\
\text { exposures } \\
\text { (SE) }\end{array}$ \\
\hline T. platneri & $17.0(3.1)_{\mathrm{i}}$ & $9.0(1.6) \mathrm{a}$ & $26.0(2.8)$ \\
T. minutum (Rv) & $14.6(3.4) \mathrm{A}$ & $10.9(2.9) \mathrm{A}$ & $23.5(4.5)$ \\
\hline
\end{tabular}

Analyses were performed separately for each Trichogramma spp. Means followed by the same letter are not significantly different (Fisher protected LSD, $P>0.05$ ).

${ }^{a} T$. minutum produced at Rincon-Vitova insectary (Ventura, CA).

both exposures on 24-h-old hosts was similar to that of the 1 exposure plus an inexperienced female. Therefore, egg depletion does not explain the lower parasitism rates observed with ovipositionally experienced females.

A 2nd explanation is that host marking occurred by the 1st female and only experienced females detect or respond to it. Both external and internal host marking by Trichogramma spp. have been reported and can prevent conspecific females from ovipositing in a visited host (Salt 1937, Klomp et al. 1980). Klomp et al. (1980), showed that ovipositionally experienced T. embryophagum detect host marking, but ovipositionally naive T. embryophag$u m$ do not and will oviposit into marked hosts. Their study was conducted with E. kuehniella eggs which are deposited singly. Egg masses of the obliquebanded leafroller, used in our studies, contain $\approx 200$ eggs per mass. However, our results are similar, indicating that host marking appears also to be effective on egg masses.

External host marking lasts only a few minutes, after which it has no effect (Salt 1937, Klomp et al. 1980). Under field conditions, where many minutes may pass between parasitoid visits to the same obliquebanded leafroller egg mass, this type of marking may have no effect in preventing additional parasitism. In our study, the 2 nd female was placed on the previously visited egg mass immediately after removal of the 1st female. It is unknown if the initial contact with external host marking inhibited oviposition by the 2nd female for the 24-h duration of the study, or if $24 \mathrm{~h}$ was sufficient time for this host marking to dissipate. However, even if many eggs were not parasitized by the 1st female, multiple oviposition attempts by the 2 nd female would greatly increase the probability of contacting a previously parasitized egg and the internal host marking, preventing further parasitism. As in previous assays, parasitism rates were significantly $(F=3.23$; $\mathrm{df}=5,194 ; P \leq 0.008)$ different among the shipments of parasitoids, ranging from 14.0 to 26.3 parasitized eggs per egg mass. 
Trichogramma spp. Searching Ability and the Effect of Provisioning. The ability of Trichogramma spp. to locate and parasitize hosts is influenced by the complexity of the environment and habitat in which the animal searches (Andow and Prokrym 1990). Hassan (1989) recommended that parasitoid host location experiments be conducted in the presence of plants under field or semifield conditions. In this assay, we also evaluated the effect of provisioning. Provisioning Trichogramma spp. has been shown to increase longevity, fecundity, and parasitism rates and may therefore increase parasitism performance in the field (Ashley and Gonzales 1974).

The ability of female Trichogramma spp. to search, locate, and parasitize obliquebanded leafroller egg masses in a complex environment containing apple trees will be more representative of field performance compared with assays conducted in vials. Provisioning with honey increases Trichogramma spp. longevity and fecundity, and it has been suggested that provisioned wasps may search for hosts whereas unprovisioned wasps search for food (Ashley and Gonzalez 1974). Therefore, this assay should indicate which Trichogramma spp. will perform better under field conditions and what effect provisioning will have.

No significant $(F=1.89 ; \mathrm{df}=1,71 ; P=0.174$ ) differences in parasitism rates resulted from provisioning. The number of parastized eggs in provisioned cages was $12.1 \pm 2.6$ (mean $\pm \mathrm{SE}$ ), and in nonprovisioned cages $7.1 \pm 2.6$ eggs were parasitized. T. platneri parasitized $12.8 \pm 3.4$ eggs per cage which was stastically the same $(F=3.13$; df $=1,71 ; P=0.081)$ as the $6.4 \pm 1.9$ eggs per cage parasitized by $T$. minutum.

Low parasitism levels in this assay when compared with those obtained in previous assays, probably reflects increased searching time in the complex environment (Andow and Prokrym 1990). In addition, we used the fewest females per cage that we felt would provide a measurable level of parasitism. Significant differences in parasitism rates were observed among insectary shipments, indicating variability of parasitoid quality from commercial insectaries $(F=2.86 ; \mathrm{df}=4,71 ; P=$ 0.006).

The manner in which obliquebanded leafroller egg masses were attached to apple leaves significantly affected parasitism levels by $T$. minutum (Rv) and T. platneri ( $F=5.35$; $\mathrm{df}=1,106 ; P=$ 0.01). Egg masses attached with plastic had fewer parasitized eggs $(1.33 \pm 0.9)$ compared with those attached with water $(5.7 \pm 1.3)$ or tape $(4.3 \pm 1.4)$. Inhibition of parasitism by $T$. pretiosum has been reported when shellac was used to attach eggs of the bollworm, Heliothis zea (Boddie), to foliage (Orphanides and Gonzalez 1970).

These findings are important because many studies examining inundative releases of Trichogramma spp. use eggs deposited onto wax paper which is then attached to foliage or eggs directly

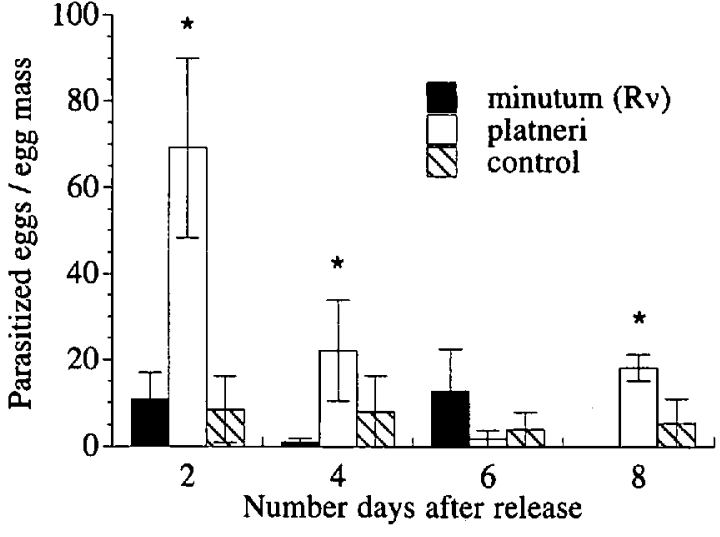

Fig. 3. Parasitism of sentinel obliquebanded leafroller egg masses in an apple orchard following inundative release of Trichogramma spp. Sentinel egg masses were collected and replaced every $2 \mathrm{~d}$ after release. *, significant treatment effects.

attached to foliage with tape or glue (Dolphin et al. 1972, Yu et al. 1984a, Andow et al. 1995). The effect these attachment methods have on searching and parasitism by natural enemies is unknown.

We speculate the plastic ring around the egg masses used in our study interfered with the ability of female Trichogramma spp. to recognize the egg mass. Trichogramma spp. use antennal drumming to perceive information about their surrounding and to locate and examine potential hosts (Schmidt and Smith 1989). Therefore, substances such as wax paper or plastic may interfere with host recognition. Wing scales, pheromones, and secretions left on the foliage and eggs during oviposition are used by parasitoids to locate hosts (Strand and Vinsion 1983, Kaiser et al. 1989). Therefore, it is possible that the use of water to attach obliquebanded leafroller egg masses to foliage still underestimates actual parasitism rates and should be examined.

Parasitism of Obliquebanded Leafroller Egg Masses by Imundatively Released Trichogramma spp. Approximately 21,500 Trichogramma were released during each trial. Of these, $57 \%$ were females, of which $13 \%$ were brachypterous. Parasitism declined significantly as time after release increased ( $F=2.89$; df $=3,35 ; P=0.049$ ) (Fig. 3). An interaction between species and day was observed ( $F=2.189 ; \mathrm{df}=6,35 ; P=0.067)$. Therefore, contrasts were constructed for each day to determine species effects. On days 2, 4, and 8, T. platneri parasitized significantly more obliquebanded leafrollers eggs per egg mass then did $T$. minutum (Rv) (day 2: $F=8.528$; $\mathrm{df}=1,35 ; P=$ 0.006 ; day $4: F=4.860$, $\mathrm{df}=1,35 ; P=0.034$; day 8: $F=10.882$; $\mathrm{df}=1,35 ; P=0.002$ ). No differences were recorded between parasitism rates in the control and $T$. minutum (Rv) release plots during any sample.

Trichogramma minutum (Rv) does not disperse $>50 \mathrm{~m}$ from the point of release (Kot 1964). Our 
treatment and control plots were separated by only $30 \mathrm{~m}$. Therefore, parasitism in control plots may have resulted from endemic Trichogramma spp. or movement of released parasitoids into these plots (Fig. 3). Although endemic Trichogramma spp. may have parasitized some egg masses in this study, the significant increase in parasitism rates in the $T$. platneri plots compared with the control is attributable to the inundative releases.

The decline in parasitism after day 2 may have resulted from parasitoids moving out of release plots. Additionally, parasitoids were allowed to emerge for $24 \mathrm{~h}$ in the growth chamber before being placed in the field. This probably resulted in large numbers of parasitoids emerging from release containers immediately upon placement in the field with little emergence occurring during subsequent days. Mixed-age parasitoid pupae have been used in field releases to maintain a constant emergence rate over time (Andow et al. 1995). Egg cards containing mixed-aged parasitoids are not commercially available, but placing parasitoids in the field every few days would have the same effect.

The lack of qualified persons to identify Trichogramma spp. precluded us from identifying species that emerged from sentinel egg masses in this study. However, it was the goal of this study to determine if inundative releases of Trichogramma spp. could be used to increase parasitism of obliquebanded leafroller egg masses under field conditions, rather than to determine what Trichogramma spp. parasitized them. We did not attempt to relate environmental conditions to Trichogramma spp. performance, because the goal of this research was to obtain baseline data on obliquebanded leafroller egg mass parasitism rates from inundative releases of Trichogramma spp. Each release was treated as a blocking factor in our experimental design, and variability caused by different conditions was controlled for with this design.

Although laboratory results do not always predict accurately the outcome of field trials (Smith et al. 1986), our laboratory results did indicate that T. platneri would parasitize more obliquebanded leafroller egg masses than $T$. minutum (Rv). However, the magnitude of difference between parasitism rates caused by the 2 species in the field was not indicated by our assays. Field performance is probably influenced by factors that are not easily evaluated in laboratory assays such as environmental conditions and plant structure. Therefore, laboratory assays should be restricted to evaluating host acceptance and suitability. We assume that Trichogramma spp. that do not accept hosts under these conditions will not parasitize the same host under field conditions. Trichogramma spp. that show a high level of host acceptance in laboratory assays should then be released into small field plots and their performance evaluated. This procedure will eliminate all but $I$ assay, and Trichogramma spp. performance will be recorded under real field conditions which are difficult if not impossible to mimic in laboratory assays.

To further our knowledge of how to manipulate inundative releases of Trichogramma in apple orchards to aid in the control of obliquebanded leafrollers, we need to examine factors that can be modified in the field to influence parasitism. In the current study we released only 1 density of parasitoids from 1 canopy location; the effect of modifying these are unknown. Increased parasitism of spruce budworm egg masses has occurred by releasing more T. minutum, but the relationship was not linear (Smith et al. 1986). Releases of T. minutum into apple at different canopy positions had no effect on the location of parasitized codling moth eggs, which were parasitized to the greatest extent near the bottom of the tree (Yu et al. 1984b). However, Zhilyaeva et al. (1975) reports $T$. minutum colonized apple trees uniformly. On balsam fir, Abies balsamea L., T. minutum caused the greatest level of spruce budworm egg parasitism in the upper canopy (Smith et al. 1986, Smith 1988). These variable results indicate the need to study each Trichogramma spp. in the environment of the targeted host.

\section{Acknowledgment}

We thank Cindy Smith (Cornell University) for maintaining the obliquebanded leafroller colonies used during this study.

\section{References Cited}

Abacus Concepts. 1989. SuperANOVA. Abacus Concepts, Berkeley, CA.

Abbott, W. S. 1925. A method of computing the effectiveness of an insecticide. J. Econ. Entomol. 18: 265-267.

Andow, D. A., and D. R. Prokrym. 1990. Plant structural complexity and host-finding by a parasitoid. Oecologia 82: 162-165.

Andow, D. A., G. C. Klacan, D. Bach, and T. C. Leahy. 1995. Limitations of Trichogramma nubilale (Hymenoptera: Trichogrammatidae) as an inundative biological control of Ostrinia nubilalis (Lepidoptera: Crambidae). Environ. Entomol. 24: 1352-1357.

Ashley, T. R., and D. Gonzalez. 1974. Effect of various food substances on longevity and fecundity of Trichogramma. Environ. Entomol. 3: 169-171.

Bai, B. B., and S. M. Smith. 1994. Pattems of host exploitation by the parasitoid wasp Trichogramma minutum (Hymenoptera: Trichogrammatidae) when attacking eggs of the spruce budworm (Lepidoptera: Tortricidae) in Canadian forests. Ann. Entomol. Soc. Am. 87: 546-553.

Chapman, P. J., and S. E. Lienk. 1971. Tortricid fauna of apple in New York. N.Y. State Agricultural Experiment Station. Genera Spec. Publ. 87-90.

Dolphin, R. E., M. L. Cleveland, T. E. Mouzin, and R. K. Morrison. 1972. Releases of Trichogramma minutum and $T$. cacocciae in an apple orchard and the effects on populations of codling moths. Environ. Entomol. 1: $481-484$. 
Glass, E. H., and G.E.R. Hervey. 1962. Continuous rearing of the red-banded leafroller, Argyrotaenia veLutinana. J. Econ. Entomol. 55: 336-340.

Hassan, S. A. 1989. Selection of suitable Trichogramma stains to control the codling moth Cydia pomonella and the two summer fruit tortrix moths Adoxopliyes orana, Pandemis heparana [Lep.: Tortricidae]. Entomophaga 34: 19-27.

1990. A simple method to select effective Trichogramma strains for use in biological control, pp. 210-204. In E. Wajnberg and S. B. Vinson [eds.], Trichogram$\mathrm{ma}$ and other egg parasitoids. INRA, Paris 1991 (Les Colloques $n^{\circ} 56$ ).

1994. Strategies to select Trichogramma species for use in biological control, pp. 54-71. In E. Wajnberg and S. A. Hassan [eds.], Biological control with egg parasitoids. CAB International. Biddles Ltd. Guildford and King's Lynn, Great Britain.

Houseweart, M. W., S. G. Southard, and D. T. Jennings. 1982. Availability and acceptability of spruce budworm eggs to parasitism by the egg parasitoid Trichogramma minutum (Hymenoptera: Trichogrammatidae). Can. Entomol. 114: 657-666.

Houseweart, M. W., D. T. Jennings, C. Welty, and S. G. Southard. 1983. Progeny production by Trichogramma minutum (Hymenoptera: Trichogrammatidae) utilizing eggs of Choristoneura fumiferana (Lepidoptera: Tortricidae) and Sitotroga cerealella (Lepidoptera: Gelechiidae). Can. Entomol. 115: 1245-1252.

Kaiser, L. M., H. Pham-Delegue, E. Bakchine, and C. Masson. 1989. Olfactory responses of Trichogramma maidis Pint.: effect of chemical cues and behavioral plasticity. J. Insect Behav. 2: 701-712.

Klomp, H., B. J. Teerink, and W. C. Ma. 1980. Discrimination between parasitized and unparasitized hosts in the egg parasite Trichogramma embryophagum (Hym., Trichogrammatidae): a matter of learning and forgetting. Neth. J. Zool. 30: 254-277.

Kot, J. 1964. Experiments in the biology and ecology of species of the genus Trichogramma Westw. and their use in plant protection. Ekol. Pol. Ser. A 12: 159.

Lawson, D. S., W. H. Reissig, A. M. Agnello, J. P. Nyrop, and W. L. Roelofs. 1996. Interference with the mate-finding communication of the obliquebanded leafroller (Lepidoptera: Tortricidae) using synthetic sex pheromones. Environ. Entomol. 25: 895-905.

Manweiller, S. A. 1986. Developmental and ecological comparisons of Trichogramma minutum and Trichogramma platneri (Hymenoptera: Trichogrammatidae). Pan-Pac Entomol. 62: 128-139.

Newton, P. J. 1993. Increasing the use of trichogrammatids in insect pest management: a case study from the forests of Canada. Pestic. Sci. 37: 381-386.
Orphanides, G. M., and D. Gonzales. 1970. Effects of adhesive materials and host location on parasitization by a uniparential race of Trichogramma pretiosum. J. Econ. Entomol. 63: 1891-1897.

Pak, G. A. 1986. Behavioral variations among strains of Trichogramma spp.: a review of the literature on host-age selection. J. Appl. Entomol. 101: 55-64.

Ruberson, J. R., M. J. Tauber, and C. A. Tauber. 1987. Biotypes of Edovum puttleri (Hymenoptera: Eulophidae) responses to developing eggs of the Colorado potato beetle (Coleoptera: Chrysomelidae). Ann. Entomol. Soc. Am. 80: 451-455.

Salt, G. 1937. The sense used by Trichogramma to distinguish between parasitized and unparasitized hosts. Proc. R. Soc., Lond. 122: 57-75.

Schmidt, J. M. 1994. Host recognition and acceptance by Trichogramma, pp. 165-200. In E. Wajnberg and S. A. Hassan [eds.], Biological control with egg parasitoids. Biddles London.

Schmidt, J. M., and J.J.B. Smith, 1989. Host exatmination walk and oviposition site selection of Trichogramma minutum: studies on spherical hosts. J. Insect Behav. 2: 143-171.

Smith, S. M. 1988. Pattem of attack on spruce budworm egg masses by Trichogramma minutum (Hymenoptera: Trichogrammatidae) released in forest stands. Environ. Entomol. 17: 1009-1015.

Smith, S. M., M. Hubbes, and J. R. Carrow. 1986. Factors affecting inundative releases of Trichogramma minutum Ril, against the spruce budworm, pp. 29-39. Symposium on Trichogramma and other egg parasites, XVII International Congress of Entomology, Hamburg, FRG.

Strand, M. R., and S. B. Vinsion. 1983. Analyses of an egg recognition kairomone of Telenomus heliothidis (Hym: Scelionidae). Isolation and function. J. Chem. Ecol. 9: 423-432.

Yu, D.S.K, E.A.C. Hagley, and J. E. Laing. 1984a. Biology of Trichogramma minutum Riley collected from apples in southern Ontario. Environ. Entomol. 13: 1324-1329.

Yu, D.S.K, J. E. Laing, and E.A.C. Hagley. 1984 b. Dispersal of Trichogramma spp. (Hymenoptera: Trichogrammatidae) in an apple orchard after inundative releases. Environ. Entomol. 13: 371-374.

Zhilyaeva, V. M., V. I. Pilipyuk, and V. A. Shchepetil'nikova. 1975. Influence of behavior of Trichogramma on its parasitism of eggs of the codling moth in the crown of apple trees, Pp. 52-55. In V. P. Pristavko [ed.], Insect behavior as a basis for developing control measures against pests of field crops and forest. Naukova Dumka, Kiev.

Received for publication 16 April 1996; accepted 13 December 1996 